\title{
The Ligand Shell as an Energy Barrier in Surface Reactions on Transition Metal Nanoparticles
}

\author{
Jeremy G. Smith ${ }^{1}$ and Prashant K. Jain ${ }^{1,2,3 *}$ \\ ${ }^{1}$ Department of Chemistry, University of Illinois, Urbana Champaign, IL 61801 USA \\ ${ }^{2}$ Department of Physics, University of Illinois, Urbana Champaign, IL 61801 USA \\ ${ }^{3}$ Materials Research Lab, University of Illinois, Urbana Champaign, IL 61801 USA \\ *Corresponding Author E-mail: jain@illinois.edu
}

SUPPORTING INFORMATION 


\section{Reagents and Instrumentation}

PELCO $^{\circledR}$ Nanoexact 30, 40, and $50 \mathrm{~nm} \mathrm{Ag} \mathrm{colloid} \mathrm{was} \mathrm{purchased} \mathrm{from} \mathrm{Ted} \mathrm{Pella} \mathrm{Inc.} \mathrm{These}$ nanoparticles have an average diameter of $25.51+/-6.28,34.37+/-5.99$ and $46.58+/-8.35 \mathrm{~nm}$ and are roughly spherical as found by transmission electron microscopy (TEM) shown in Figure S13. These nanospheres, coated by trisodium citrate $\left(\mathrm{Na}_{3} \mathrm{Citrate}\right)$ molecules, were suspended in 2 $\mathrm{mM} \mathrm{Na} \mathrm{N}_{3}$ Citrate buffer.

Ag nanoplates were synthesized according to a procedure developed in our laboratory. To a 20 $\mathrm{mL}$ scintillation vial containing $10 \mathrm{~mL}$ of deionized (DI) water (18.2 $\mathrm{M} \Omega$, Barnstead Nanopure), $25 \mu \mathrm{L}$ of $100 \mathrm{mM} \mathrm{AgNO}_{3}($ Amresco, 99.0\%) and $25 \mu \mathrm{L}$ of $100 \mathrm{mM}$ trisodium citrate (Alfa Aesar, $99.0 \%$ ) were added. This solution was heated to $95{ }^{\circ} \mathrm{C}$, in air, with stirring. $100 \mu \mathrm{L}$ of 20 mM sodium borohydride (J.T. Baker, 98\%) was rapidly injected. The solution immediately turned yellow and remained that color. If the reaction temperature was too low, agglomerated nanoparticles were formed, indicated by a solution color of brown or black. Heating was maintained for $1 \mathrm{~min}$, and then the solution was allowed to cool to room temperature. After 30 min, this solution of seed particles was stored in a refrigerator at $4{ }^{\circ} \mathrm{C}$ without purification. The seed solution stored in this manner is capable of being used for synthesis of high quality nanoparticles for at least 6 months.

In a $20-\mathrm{mL}$ scintillation vial containing $10 \mathrm{~mL}$ of DI water $(18.2 \mathrm{M} \Omega), 25 \mu \mathrm{L}$ of $100 \mathrm{mM} \mathrm{AgNO}_{3}$ and $15 \mu \mathrm{L}$ of $100 \mathrm{mM}$ trisodium citrate were added. To this solution, $50-400 \mu \mathrm{L}$ of the seed solution was added. Adding a smaller amount of seed solution resulted in larger nanoparticles, and red shifted spectra. With stirring, under air at room temperature, $15 \mu \mathrm{L}$ of $100 \mathrm{mM} \mathrm{L-(+)-}$ ascorbic acid (Alfa Aesar, 99\%) was rapidly injected. The solution gradually changed color from 
faint yellow, to red, to purple, to blue. Following 30 min of stirring, the vial was removed and stored in the fridge. The solution was found to be stable over several months. All experiments for the current investigation were done with nanoparticles made with $300 \mu \mathrm{L}$ of the seed solution. After $30 \mathrm{~min}$ of stirring, the nanoplates were centrifuged at $11,000 \mathrm{rpm}$ for $10 \mathrm{~min}$. The pellet was collected and resuspended in $10 \mathrm{~mL}$ of $2 \mathrm{mM} \mathrm{Na}_{3}$ Citrate and stored wrapped in foil in a fridge prior to experiments. The sample was found to be crystalline nanoplates, with stacking faults perpendicular to the flat face, ranging from hexagonal to prismatic in shape (Figure S13). The large flat face was found to comprise the (111) facet of Ag, with an average face diameter of $37.24+/-9.36 \mathrm{~nm}$ and a thickness of $5.25+/-0.78 \mathrm{~nm}$. $\mathrm{Au}(\mathrm{III}) \mathrm{Cl}_{3}$ was purchased from Sigma Aldrich $(99 \%)$ and used without further purification. Deionized water (18.2M $\Omega$ ) from a Barnstead Nanopure water system was used. Transmission electron microscopy (TEM) was performed on a JEOL 2100 operating at 200kV. 3-mercaptopropionic acid (3-MPA, 99\%), 6mercaptohexanoic acid (6-MHA, 90\%), and 11-mercaptoundecanoic acid (11-MUA, 99\%) were all purchased from Sigma Aldrich. $\mathrm{AgNO}_{3}$ was purchased from Amresco, (99.0\%), $\mathrm{Na}_{3}$ Citrate was purchased from Alfa Aesar, (99.0\%), sodium borohydride from J.T. Baker, (98\%), and L(+)-ascorbic acid from Alfa Aesar, (99\%).

\section{Flow Cell Preparation}

All single-nanoparticle experiments were carried out in home-built flow cells amenable to darkfield scattering microscopy. Flow cells were prepared immediately prior to use. A hole was drilled in a glass slide ( 3 inch $\mathrm{x} 1$ inch $\mathrm{x} 1$ inch, VWR Vistavision) using a 1-mm diamond coated drill bit operating at $\sim 20 \mathrm{kRPM}$ under water. Using a 2.5 -mm diamond coated drill at $20 \mathrm{kRPM}$, a second hole was drilled $\sim 50 \mathrm{~mm}$ from the first hole to serve as an outlet. Slides were cleaned by immersion in $2 \mathrm{M} \mathrm{KOH}$ for $30 \mathrm{~min}$ at $\sim 85^{\circ} \mathrm{C}$, thoroughly rinsed with deionized (DI) water, 
rinsed with saturated $\mathrm{NaHCO}_{3}$, sonicated for $10 \mathrm{~min}$ in DI water, rinsed again thoroughly with DI water, sonicated in DI water for another $30 \mathrm{~min}$, and then rinsed again with DI water, before blow drying with pressurized air. Coverslips ( 24 x $60 \mathrm{~mm}$, No.1, VWR Superslip) were subjected to the same cleaning procedure. Nanoparticles were cast onto the cleaned coverslips immediately after drying. A drop (from a plastic pipette) of the undiluted Ag colloid solution was added to 2 drops of DI water in an Eppendorf tube and mixed. This solution was then drop cast onto the cleaned coverslip, and spread across the entire surface of the coverslip by tilting the coverslip. Since the coverslips are freshly cleaned, the solution wets the surface with ease. The excess liquid is wicked off using a paper towel, leaving a thin layer of liquid on the surface. This thin layer is then blown off with pressurized air. This is found to leave behind a uniform coating of particles, sufficiently dispersed to allow single-nano particle spectroscopy/microscopy. Polyethylene tubing (Instech Laboratories, $.076 \mathrm{~mm}$ inner diameter) was inserted into the $1-\mathrm{mm}$ drilled hole and glued into place with 5 minute-epoxy (Loctite) and allowed to cure for $15 \mathrm{~min}$. Double sided tape (3M) placed along each long-edge of the slide defined the height of the flow channel (tens of $\mu \mathrm{m}$ ). Epoxy was applied around the interior edges of the tape to create a liquidtight seal and the nanoparticle coated coverslip was placed on top of the tape and gently pushed down so that the epoxy spread evenly. The flow cell was then allowed to dry for $30 \mathrm{~min}$ before imaging experiments were performed.

$\mathrm{Na}_{3}$ Citrate on the nanoparticle surface was exchanged by one of the thiols: 3-MPA, 6-MHA, or 11-MUA in the following manner. After the epoxy had dried, the flow cells were purged with 2 $\mathrm{mL}$ of DI water, followed by $2 \mathrm{~mL}$ of EtOH. Following this, $2 \mathrm{~mL}$ of a $5 \mathrm{mM}$ solution of the thiol in 50:50 mixtures of DI water and EtOH was flowed through the cell and allowed to sit for 15 min. Following this, the flow cell was purged with $3 \mathrm{~mL}$ of EtOH (to remove the excess ligand) 
and then $3 \mathrm{~mL}$ of DI water (to remove the ethanol). For experiments with $\mathrm{Na}_{3} \mathrm{Citrate}$ covered nanoparticles, the flow cells were first purged with $2 \mathrm{~mL}$ of DI water, followed by purging with 5 $\mathrm{mM} \mathrm{Na}_{3}$ Citrate in DI water, allowed to sit for $15 \mathrm{~min}$, then purged with $3 \mathrm{~mL}$ of DI water. From separate work in our laboratory, ${ }^{1}$ we know 15 min to be sufficient time for obtaining near saturation coverages of thiols on Ag nanoparticles.

Each day of the experiment, a $1 \mathrm{mM}$ stock solution of $\mathrm{Au}(\mathrm{III}) \mathrm{Cl}_{3}$ was prepared in DI water. This solution was kept wrapped in foil, in the refrigerator between experiments. The concentration of $\mathrm{Au}(\mathrm{III}) \mathrm{Cl}_{3}$ was confirmed by taking UV-Vis spectra of these stock solutions. These stock solutions were then diluted to $\mu \mathrm{M}$ concentrations immediately prior to every experiment. The solutions were kept wrapped in foil. Following a purge of the flow cells with EtOH and DI water, a $3 \mathrm{~mL}$ plastic syringe containing a solution of freshly prepared $\mathrm{Au}(\mathrm{III}) \mathrm{Cl}_{3}$ was affixed into the inlet tubing of the flow cell. This was done in such a way as to leave a small bubble between the solvent filling the flow cell and the $\mathrm{Au}(\mathrm{III}) \mathrm{Cl}_{3}$ solution. The bubble prevented mixing of the $\mathrm{Au}(\mathrm{III}) \mathrm{Cl}_{3}$ solution and the solvent already present in the flow cell. Entry of this bubble into the flow cell was used to define $\mathrm{t}=0 \mathrm{~s}$.

\section{Single-Nanoparticle Microscopy}

In-situ microscopy was performed in two modes: imaging and spectroscopy. In-situ imaging was performed on an Olympus IX-71 microscope equipped with an Andor iXon DU897E backviewed electron-multiplying charge-coupled device (CCD) camera. In-situ spectroscopy was performed on an Olympus IX-51 microscope equipped with an Acton SP 2300 spectrograph with a 300 blaze grating and a liquid nitrogen-cooled Princeton Instruments PyLoN CCD camera. 
For both studies, Ag nanoparticles were immobilized on the flow cell surface and illuminated with a Olympus U-LH100-3 100W halogen lamp source focused through an Olympus U-DCW 1.2-1.4 NA oil immersion dark field condenser. Scattering from the sample was collected by an Olympus UPlanApo 0.5-1.35 NA 100x oil immersion objective. It was ensured that individual nanoparticles were well separated from one other, such that they could be addressed individually, despite the diffraction-limited nature of the microscopy. The flow cell was placed on top of the objective, with the coverslip facing down towards the objective. The objective height was set to focus on the plane of the immobilized nanoparticles, following which the condenser height was adjusted so as to also focus on the sample plane. Next, the objective iris was adjusted to maximize the signal-to-noise ratio of the dark-field scattering signal. An iris between the lamp source and the condenser was adjusted to illuminate an area somewhat larger than the field of view of the microscope eyepiece, which in turn is $\sim 4 \mathrm{x}$ larger than the field of the CCD camera. The outlet of the flow cell was covered with a Kimwipe to absorb the outflowing liquid. The syringe containing the $\mathrm{Au}(\mathrm{III}) \mathrm{Cl}_{3}$ solution was placed on a $\mathrm{KD}$ Scientific syringe pump operating at a flow rate of $1.5 \mathrm{~mL} / \mathrm{hr}$. The point when the bubble entered the cell was set to $\mathrm{t}=0 \mathrm{~s}$.

Real-time intensity-only images were acquired for a wide-field of nanoparticles on the Andor Ixon+ EMCCD, with its acquisition time set to $0.5,1,1.5$, or $2 \mathrm{~s}$ and gain set to zero. Experiments performed at the two highest powers (100 and 45.93\%) on the $34 \mathrm{~nm}$ nanospheres utilized a $0.5 \mathrm{~s}$ acquisition time, the third highest power (17.78\%) used a $1 \mathrm{~s}$ acquisition time, and the lowest power (12.11\%) utilized a $2 \mathrm{~s}$ acquisition time. For experiments performed on 26 $\mathrm{nm}$ nanospheres, the acquisition time was set to $1.5 \mathrm{~s}$ with the lamp power set to a maximum, in order to account for the relatively lower scattering intensity of nanospheres of this size. For 47 $\mathrm{nm}$ nanospheres, the lamp power was set to $33.41 \%$ of the maximum fluency setting and a $0.5 \mathrm{~s}$ 
acquisition time was used. For experiments performed on nanoplates, the lamp power was set to a maximum and the acquisition time was $0.5 \mathrm{~s}$.

In another case (Figure S4), real-time spectral images were obtained for several single nanoparticles. The lamp intensity was set to a maximum and the acquisition time was set to $2 \mathrm{~s}$. Individual nanoparticles were aligned within the slit of the spectrograph. Acquired spectra were corrected during collection in Winspec software by subtraction of dark count signal and division by the light source spectrum.

\section{Single-Nanoparticle Data Processing and Analysis}

The time-stacked intensity images were processed using Image J. First, the images were corrected for stage drift using a manual image registration algorithm in Image J. Next, an area surrounding each individual nanoparticle, such that no interference from other nanoparticles was present, was marked. The intensity of this selected area was extracted using the Image J multimeasure tool for the entire time stack. In this way, time-trajectories of intensity were obtained for several individual nanoparticles in each experiment. The single-nanoparticle intensity trajectories were fit to a sigmoid function of the Boltzmann form in Origin:

$$
y=\frac{A_{1}-A_{2}}{1+e^{\frac{\left(t-x_{c}\right)}{\tau}}}+A_{2}
$$

where fit parameter $A_{1}$ is the intensity at the start of the trajectory and $A_{2}$ is the intensity at the end of the trajectory. $x_{c}$, the instant of time relative to the start of flow where the intensity crosses a half-way point between $A_{1}$ and $A_{2}$, defines the waiting time for an individual nanoparticle. The time constant $\tau$, obtained from the fit (Figure S1), represents a characteristic time for an 
individual nanoparticle to undergo the abrupt conversion. The ensemble trajectory is obtained by averaging all single-nanoparticle trajectories in a given experiment.

Time-stacked spectral images were also corrected for stage-drift in Image $\mathrm{J}$ and then converted into scattering spectra as a function of time for each individual nanoparticle in the experiment. At each time point, the peak maximum of the scattering spectrum, $\lambda_{\max }$, was obtained by fitting the spectrum to a single Lorentzian function in Origin software. In a separate analysis, the integrated signal under the acquired raw spectrum (obtained by multiplying the corrected scattering spectrum by the lamp spectrum) was plotted as a function of time to generate time-trajectories of intensity for single nanoparticles. The latter procedure was performed to check for correlation between spectral shifts that individual nanoparticles undergo during galvanic exchange with changes in the scattering intensity occurring during the process (Figure S4).

Histograms of waiting times were, in all cases, fit to Gaussian distributions (Figure S2). From this fit, the peak maximum was defined as the average waiting time, and the full-width-at-halfmaximum (FWHM) was used to define an ensemble reaction time constant, which is the inverse of the ensemble reaction rate constant. It is important to note that in generating the histograms, all of the fit values were not used. Several single-nanoparticle trajectories behave spuriously due to a variety of potential reason including drifting of a nanoparticle outside of the imaging area, a nanoparticle de-adhering from the surface, or scattering interference from bubbles in solution. It is cumbersome to manually identify and correct the trajectories suffering from such complications. Therefore, any trajectory that yielded large errors in either of the fit parameters $(\tau$ or waiting time) was excluded from the analysis, while ensuring that a sufficiently large number of trajectories are included in the analysis for sound statistics. 
Single-nanoparticle $\tau$ distributions did not appear to be Gaussian in nature, and frequently showed tails towards longer waiting times. Therefore, a lognormal distribution was chosen to fit this data (Figure S2) and the peak of this distribution was used to obtain a peak $\tau$.

Concentration dependence of the FWHM was assumed to follow a power law of the form:

$$
\mathrm{FWHM}=a\left(\frac{c}{c_{\text {lig }}}\right)^{b}
$$

where $c$ is the $\mathrm{Au}(\mathrm{III}) \mathrm{Cl}_{3}$ concentration, $c_{\text {lig }}$ is a ligand length-dependent scaling factor, $b$ is phenomenologically related to a reaction order, and $a$ is a proportionality factor of $1 \mathrm{M}^{-\mathrm{b}} \cdot \mathrm{s}^{-1}$ for consistency of units. Log-log plots of FWHM vs. $\mathrm{Au}(\mathrm{III}) \mathrm{Cl}_{3}$ concentration, $c$ for 3-MPA, 6MHA, and 11-MUA cases were subject to a linear fit:

$$
\log (F W H M)=b * \log \left(c_{\text {lig }}\right)-b * \log (c)
$$

$c_{\text {lig }}$ was obtained from the intercept of this straight line fit as $10^{\text {intercept } b} . b$, which is the negative of the slope of the fit, was globally constrained to be the same for plots for all three ligand lengths. The latter is justified on the basis of our results of Figure 1, which show that the presence of the ligand does not change the nature of the rate-limiting step and therefore reaction order is likely to be unaltered. The fits obtained with $b$ globally constrained are show in a linearlinear form by solid curves in Figure 3 and indicate a value of $b=1.62$, which is within the range of values obtained from separate unconstrained fits for the three ligand coatings.

\section{Ensemble Solution-Phase Galvanic Exchange Experiments for Structural Characterization}

In these experiments, $34 \mathrm{~nm} \mathrm{Ag}$ nanospheres suspended in DI water and $2 \mathrm{mM} \mathrm{Na}_{3}$ Citrate were first subject to ligand exchange by adding $20 \mu \mathrm{L}$ of $200 \mu \mathrm{M}$ solutions of 3-MPA, 6-MHA, or 11- 
MUA. Ligand exchange was confirmed by the red-shift of the plasmon band in UV-Vis absorption spectra. Following ligand exchange, the nanospheres were centrifuged at 10,000 rpm for 10 min and then resuspended in DI water. The $\mathrm{Na}_{3}$ Citrate coated nanospheres were subject to the same centrifugation procedure for removal of excess ligand. Next, the nanosphere solution, in each of the four cases $\left(\mathrm{Na}_{3}\right.$ Citrate, 3-MPA, 6-MHA, and 11-MUA), was titrated with a solution of $\mathrm{Au}(\mathrm{III}) \mathrm{Cl}_{3}$ in DI water. The degree of galvanic exchange of the $\mathrm{Ag}$ nanoparticles over the course of the titration was followed by UV-Vis absorption spectrophotometry (Figure S11). At two points in the titration, samples, deemed intermediate and final (spectra shown by red and green curves respectively in Figure S11), were collected for TEM imaging and EDS analysis. Intermediate points correspond to $3,20,30$, and $30 \mathrm{nmol}$ of $\mathrm{Au}(\mathrm{III}) \mathrm{Cl}_{3}$ added, for $\mathrm{Na}_{3}$ Citrate, $3-$ MPA, 6-MHA and 11-MUA respectively. Final points correspond to 20, 45, 55, and $100 \mathrm{nmol}$ of $\mathrm{Au}(\mathrm{III}) \mathrm{Cl}_{3}$ added, for $\mathrm{Na}_{3}$ Citrate, 3-MPA, 6-MHA and 11-MUA respectively. Intermediate points were found by adding $\mathrm{Au}(\mathrm{III}) \mathrm{Cl}_{3}$ in aliquots, with vigorous mixing followed by waiting for 1 hour before a UV-Vis spectrum was acquired. Aliquot amounts were $5 \mathrm{nmol}$, except for the $\mathrm{Na}_{3}$ Citrate case, where exchange was nearly complete after addition of $5 \mathrm{nmol}$, so smaller aliquots of $3 \mathrm{nmol}$ were employed to make samples. The first point in the titration where a noticeable change was seen in the spectrum was termed the intermediate stage. The final sample in each titration was collected at the point where subsequent addition of $\mathrm{Au}(\mathrm{III}) \mathrm{Cl}_{3}$ produced no noticeable changes in the UV-Vis spectrum.

Grids for TEM imaging were prepared by drop-casting $15 \mu \mathrm{L}$ of the sample suspension onto an ultrathin carbon supported on Cu TEM grid (Ted Pella), followed by drying for 2 hours, washing with copious amounts of DI water and further dried in vacuum overnight. High-angle annular dark-field (HAADF) scanning transmission electron microscopy (STEM) imaging, and energy 
dispersive X-ray spectroscopy (EDS) analysis of the prepared samples was performed on a JEOL-2010F instrument, operating at $200 \mathrm{keV}$ and with a spot size of $0.3 \mathrm{~nm}$, an image resolution of 512 x 512 pixels, and a dwell time of $32 \mu$ s per pixel. EDS spectra were recorded on an Oxford EDS detector. A time constant of $4 \mathrm{~s}$ and a total acquisition time of $40 \mathrm{~s}$ at a magnification of 30k was used for wide-field EDS spectra acquired in brightfield (Figure 4, bottom row). Elemental mapping was performed with a $0.3 \mathrm{~nm}$ spot size in STEM, an image resolution of $75 \times 75$ pixels, a dwell time of 2 ms per pixel, a time constant of $16 \mathrm{~s}$, over 100 frames with drift correction every 3 frames. Maps shown in Figure 5 and Figure S12 are overlays, which have been down-sampled by averaging over 4 pixels. Elemental quantification was performed in the IXRF Iridium Ultra software, using the integrated intensities of the Ag and $\mathrm{Au} \mathrm{L}_{\mathrm{a}}$ lines and the $\mathrm{Cl} \mathrm{K}$ line in the EDS spectra. 

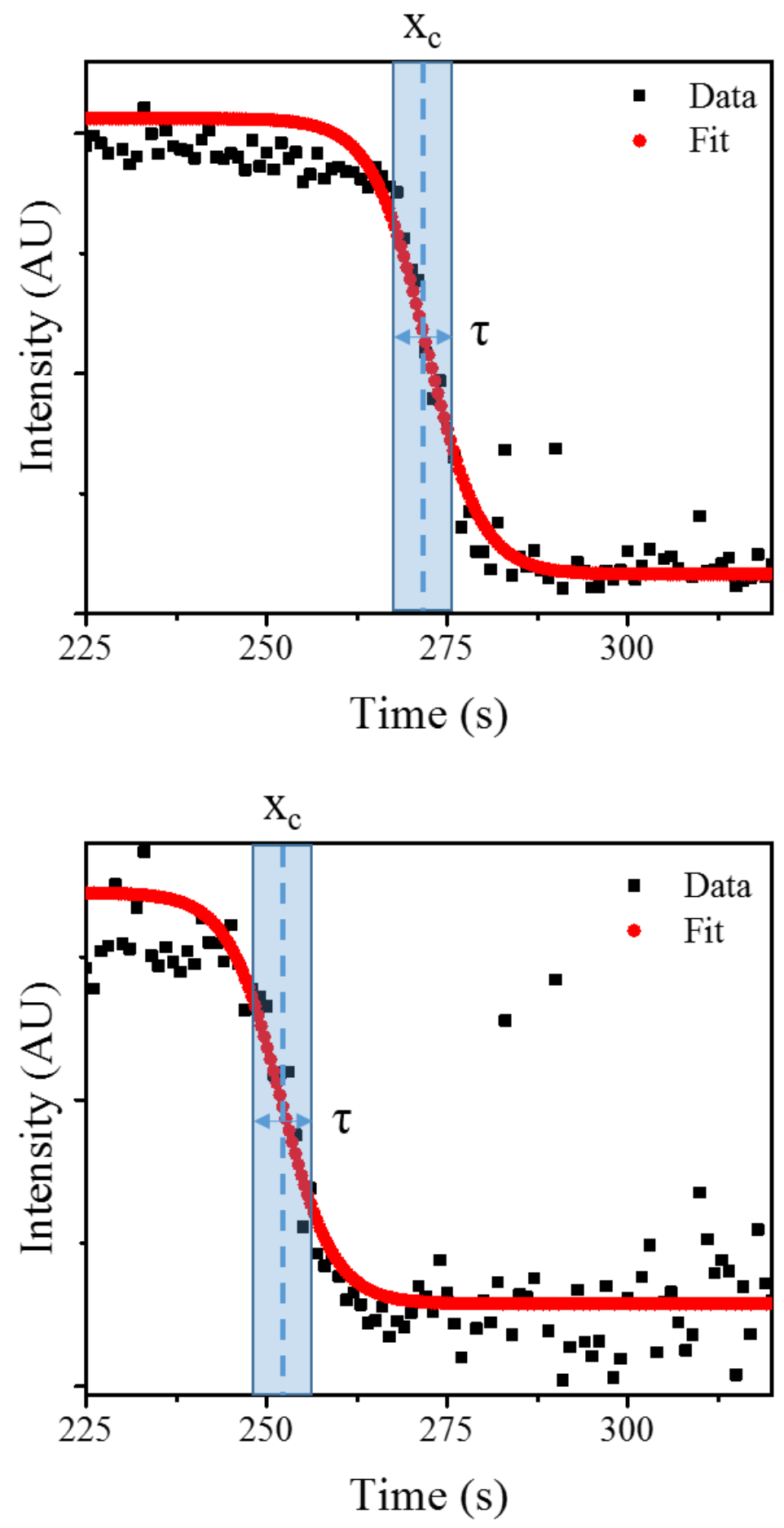

Figure S1. Two examples of single-nanoparticle trajectories and sigmoidal fits from the 3-MPA case shown in Figure 1. The waiting time $\left(\mathrm{x}_{\mathrm{c}}\right)$ is obtained from the midpoint of the sigmoid, as indicated. The single-nanoparticle time constant $(\tau)$ corresponds to half the time it takes the trajectory to go from the maximum to minimum intensity. These experiments were performed at $17.78 \%$ of the maximum power, $1.5 \mathrm{~mL} / \mathrm{hr}$ flow rate, and a $6 \mu \mathrm{M}$ concentration of $\mathrm{Au}(\mathrm{III}) \mathrm{Cl}_{3}$. 

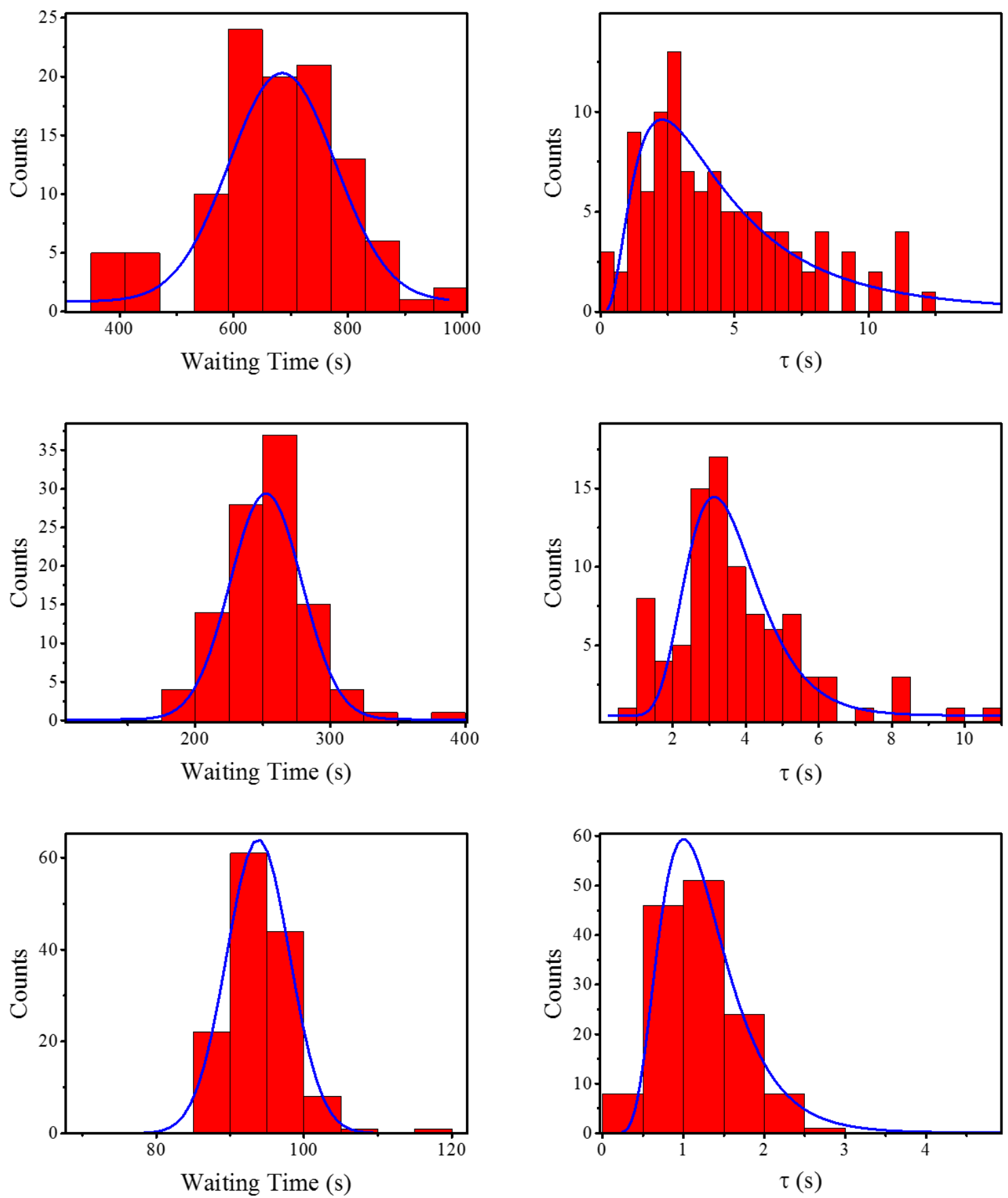

Figure S2. Examples of Gaussian fits to waiting time histograms (left) and lognormal fits to $\tau$ histograms (right) for the 6-MHA, 3-MPA and $\mathrm{Na}_{3}$ Citrate (from top to bottom) cases shown in Figure 1. Adjusted $\mathrm{R}^{2}$ values of $0.845,0.948$, and 0.999 were obtained for Gaussian fits of waiting time histograms for 6-MHA, 3-MPA, and $\mathrm{Na}_{3}$ Citrate respectively. Adjusted $\mathrm{R}^{2}$ values of $0.854,0.830$, and 0.987 were obtained for lognormal fits of $\tau$ histograms for 6-MHA, 3-MPA and $\mathrm{Na}_{3}$ Citrate respectively. 

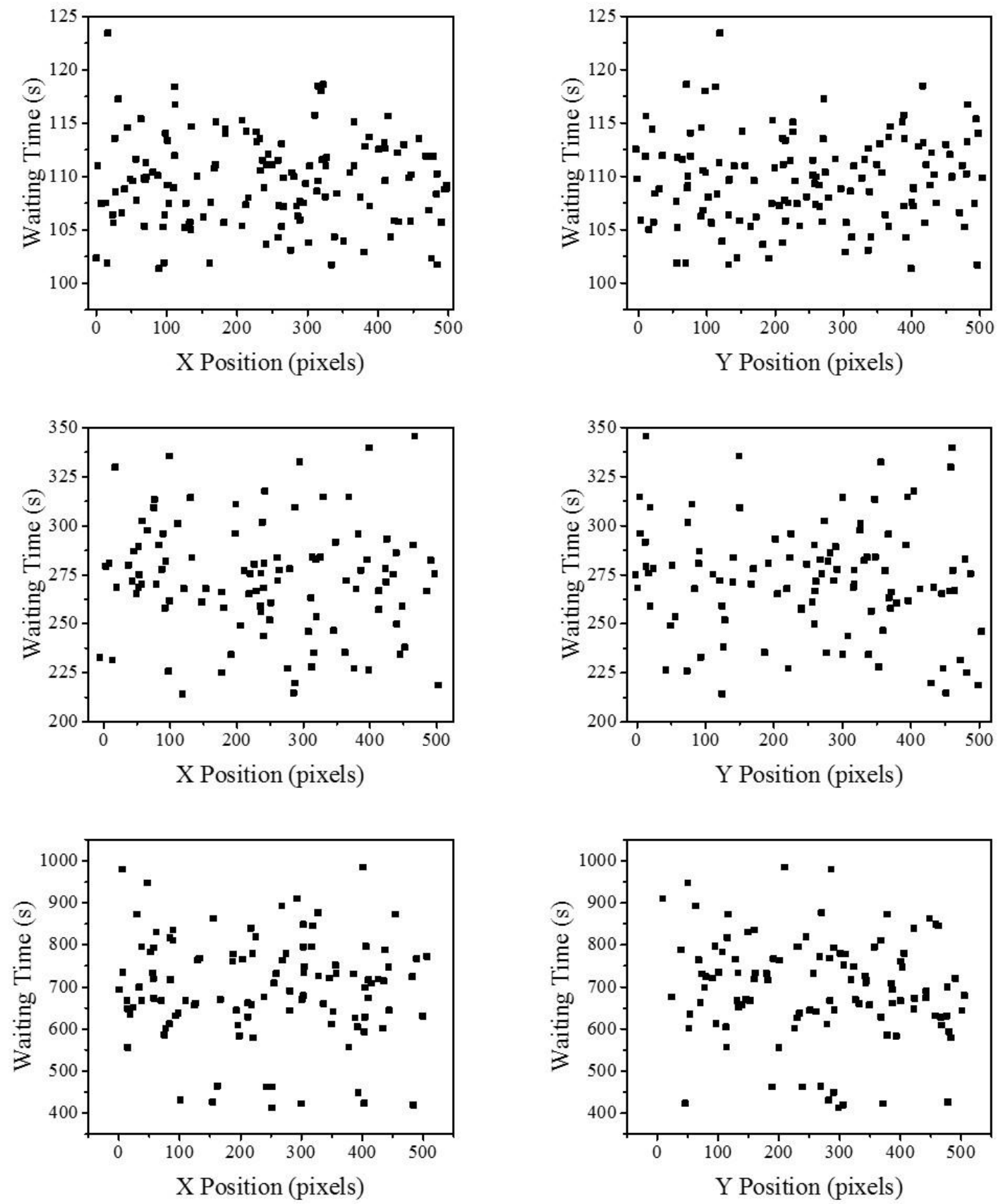

Figure S3. Plots show the lack of position bias in waiting times of individual nanoparticles for the $\mathrm{Na}_{3}$ Citrate, 3-MPA, and 6-MHA (from top to bottom) cases shown in Figure 1. For several nanoparticles in each experiment, the waiting time is plotted versus the location of the nanoparticle in the field of view, in the form of $\mathrm{X}$ and $\mathrm{Y}$ pixel positions. 

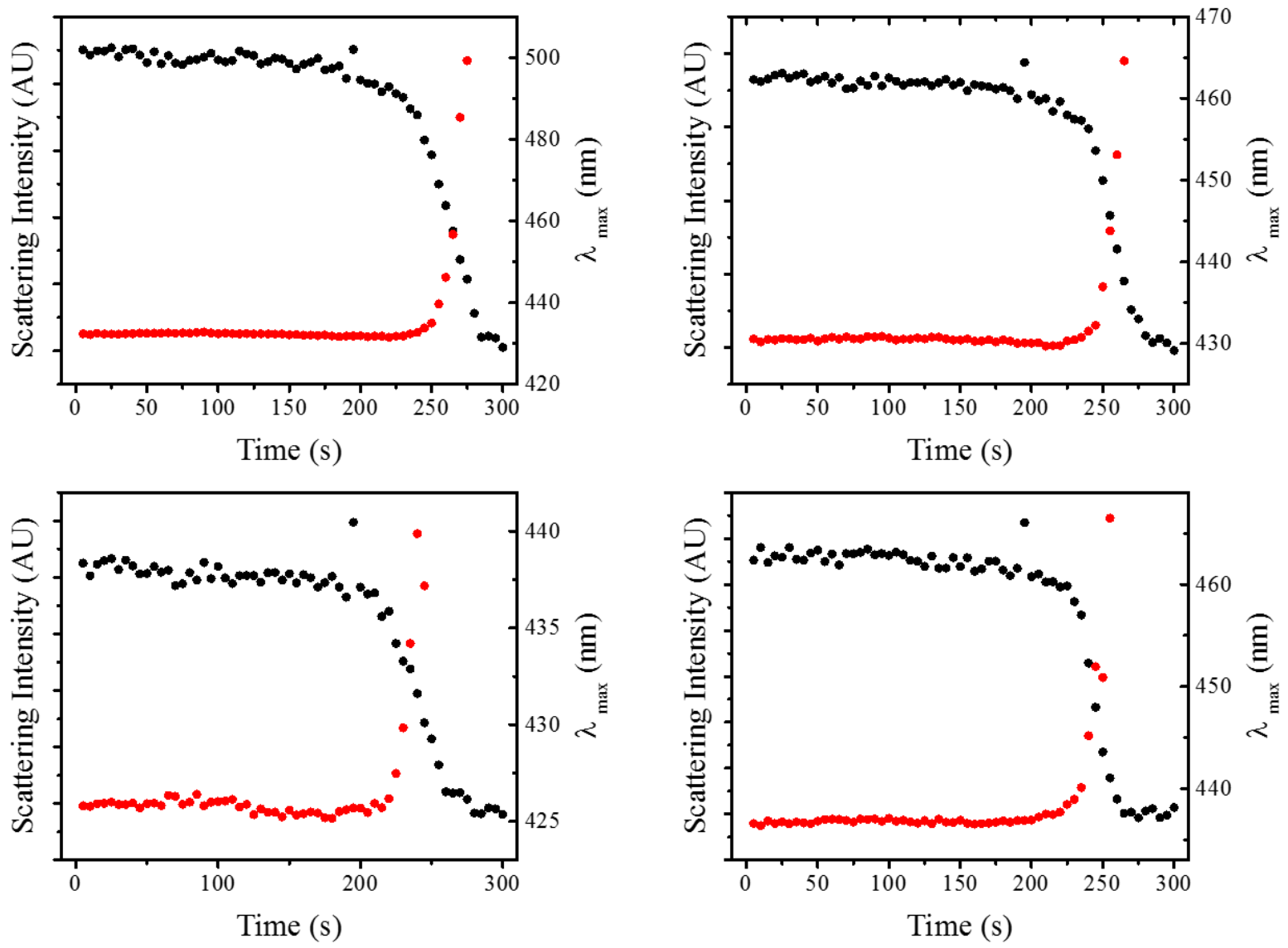

Figure S4. From single-nanoparticle spectra acquired as a function of time we show that the decrease in scattering intensity (black dots) resulting from galvanic exchange is correlated in time with the red-shift in the plasmonic scattering wavelength maximum (red dots). We show examples of such correlation for four individual Ag nanoparticles. These experiments were performed on $34 \mathrm{~nm}$ nanospheres coated with 3-MPA. We used a $\mathrm{Au}(\mathrm{III}) \mathrm{Cl}_{3}$ concentration of 2 $\mu \mathrm{M}$, the maximum lamp power, and a flow rate of $1.5 \mathrm{~mL} / \mathrm{hr}$. Scattering spectra were obtained by dividing the as-acquired spectra by the lamp spectrum. The scattering spectrum at each timepoint was fit to a single Lorentzian peak. The peak maximum, $\lambda_{\max }$ (red dots), is plotted here as a function of time. Acquisition time for collection of spectra was set to $2 \mathrm{~s}$. 


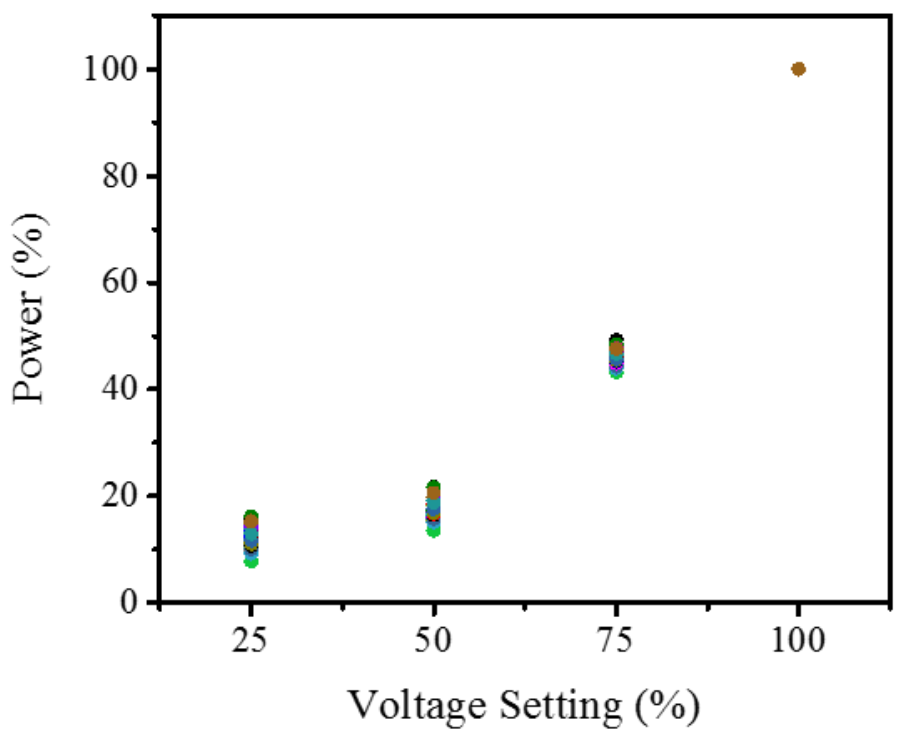

Figure S5. Relative lamp power used in the dark-field scattering experiments was calibrated to the voltage setting on the lamp. For these experiments a wide-field of 59 large $(0.5-1 \mu \mathrm{m}$ in diameter) polystyrene beads were imaged at a number of different voltage settings of the lamp. The scattering intensity was measured using the CCD, with the same integration time of $0.5 \mathrm{~s}$ at each voltage setting. The absolute scattering intensity varied from bead to bead. Therefore, a $\%$ scattering intensity was determined for each bead by normalizing the absolute intensity to the scattering intensity at the highest power. At each voltage setting, an average of the \% scattering intensity was determined from all the beads. This average \%scattering intensity obtained at each voltage setting represents the relative lamp power (\%) achieved at that voltage. 
A
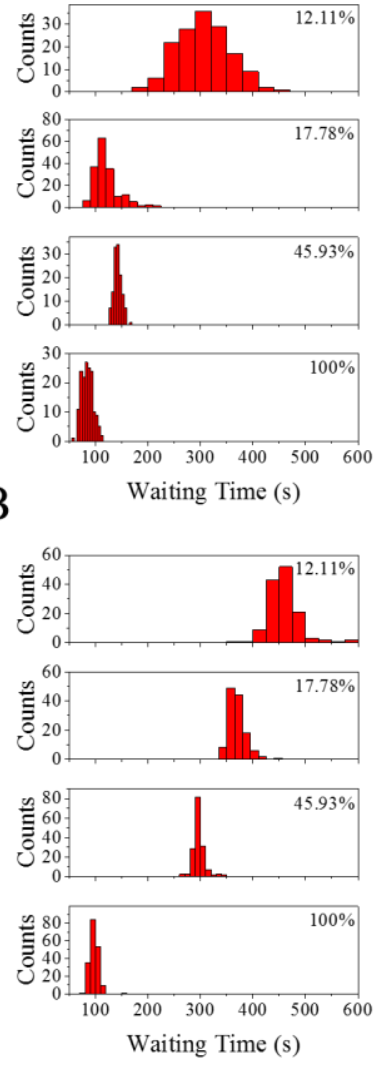
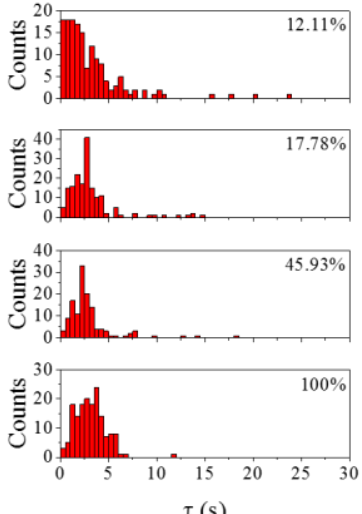

$\tau(\mathrm{s})$
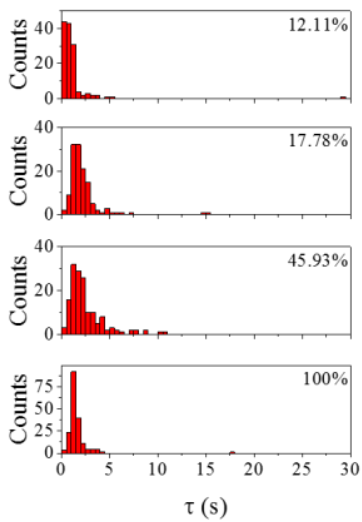

$\mathrm{C}$
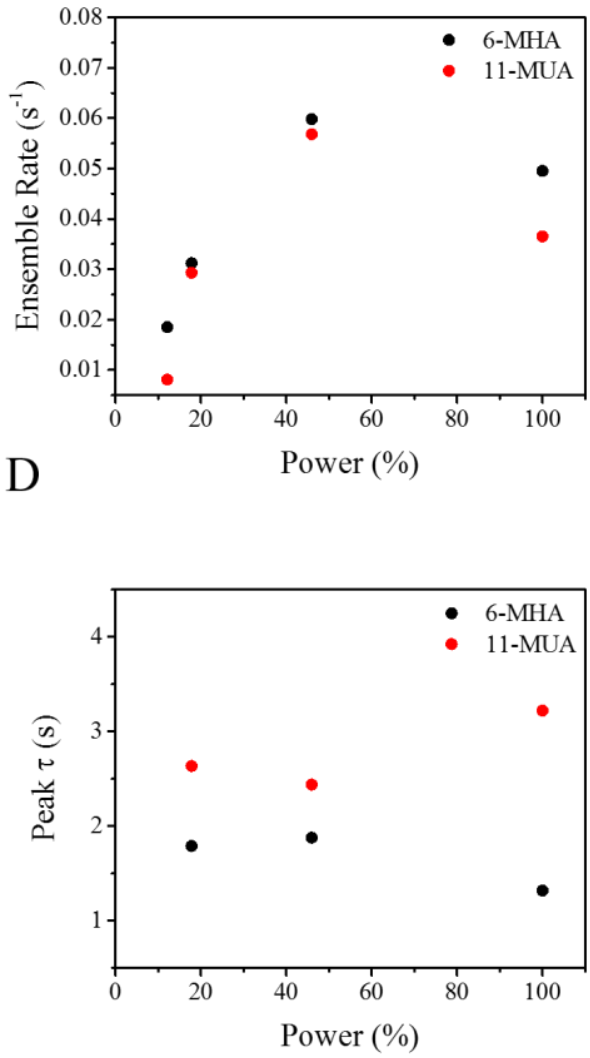

Figure S6. Effect of lamp power on the reactivity of 6-MHA and 11-MUA coated $34 \mathrm{~nm}$ nanospheres. Specifically, the waiting time (left) and $\tau$ distributions (right) are shown at four different \% lamp powers for 6-MHA (A) and 11-MUA (B) coated $34 \mathrm{~nm}$ nanospheres. Experiments with 6-MHA coeated nanospheres were performed with a $\mathrm{Au}(\mathrm{III}) \mathrm{Cl}_{3}$ concentration of $10 \mu \mathrm{M}$ and a flow rate of $1.5 \mathrm{~mL} / \mathrm{hr}$. Acquisition times of $0.5,0.5,1$ and $2 \mathrm{~s}$ were used for 100, 45.93, 17.78 and $12.11 \%$ lamp power, respectively. Experiments with 11-MUA nanospheres were performed at a $\mathrm{Au}(\mathrm{III}) \mathrm{Cl}_{3}$ concentration of $20 \mu \mathrm{M}$ and a flow rate of 1.5 $\mathrm{mL} / \mathrm{hr}$. Acquisition times of 0.5, 0.5, 1 and $2 \mathrm{~s}$ were used for 100, 45.93, 17.78 and $12.11 \%$ lamp power, respectively. For the lowest power, the $\tau$ values are likely underestimated due to the low acquisition time; however the waiting times, which range 10s of $\mathrm{s}$, are less affected by the potential error introduced by the $2 \mathrm{~s}$ integration time. The ensemble rate, i.e., the inverse of the FWHM of the waiting time distribution, (C) and the peak $\tau$ is plotted as a function of $\%$ lamp power for both 6-MHA and 11-MUA experiments. 

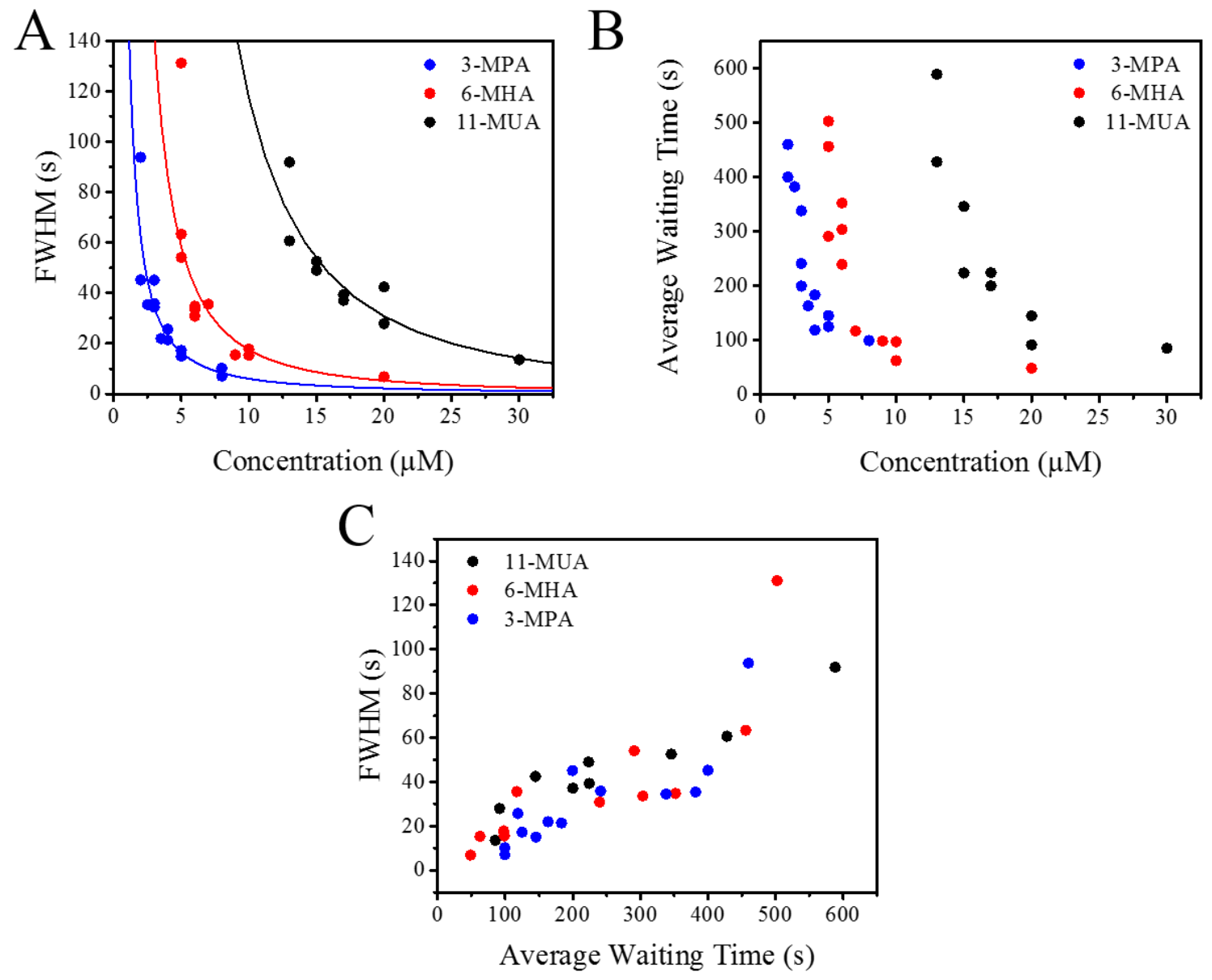

Figure S7. (A) FWHM and (B) average waiting time increase with decreasing concentration of $\mathrm{Au}(\mathrm{III}) \mathrm{Cl}_{3}$ for 3-MPA (blue), 6-MHA (red) and 11-MUA (black) coated Ag nanospheres. All experiments were performed in DI water at a flow rate of $1.5 \mathrm{~mL} / \mathrm{hr}$ and at the maximum lamp power. The average waiting time and FWHM were derived from a Gaussian fit of the waiting time distribution, as exemplified in Figure S2. We demonstrate that the average waiting time follows the same trend as a function of $\mathrm{Au}(\mathrm{III}) \mathrm{Cl}_{3}$ concentration as the FWHM. (C) We also show that there is strong correlation (Pearson's $r=0.84$ ) between the FWHM and the average waiting time values measured across all experiments. 

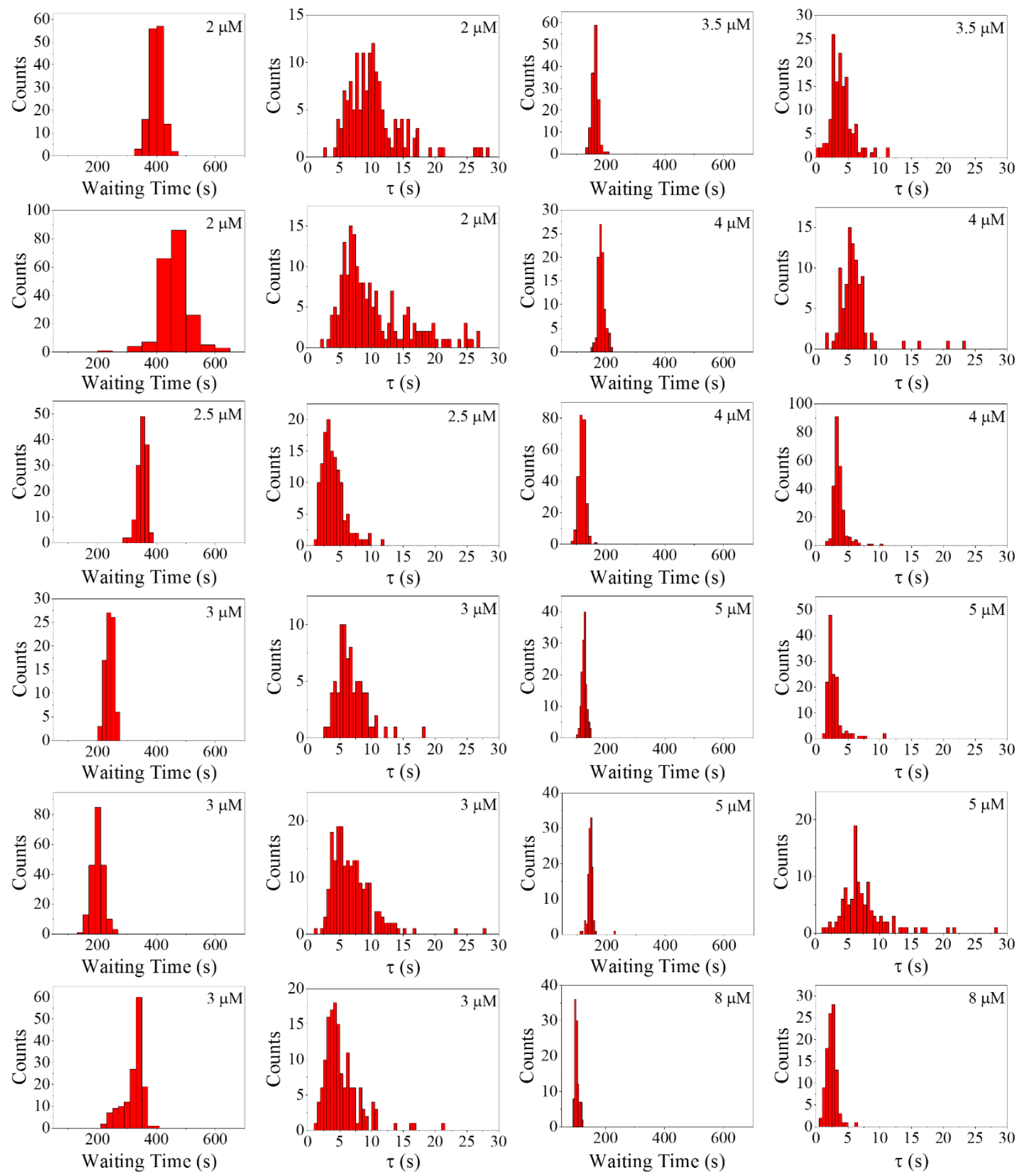

Figure S8. Histograms of waiting times and $\tau$ values for the entire set of $\mathrm{Au}(\mathrm{III}) \mathrm{Cl}_{3}$ concentration dependence experiments performed on $34 \mathrm{~nm}$ Ag nanospheres coated with 3MPA. Fits to these histograms were used to generate the data in Figure 3. 

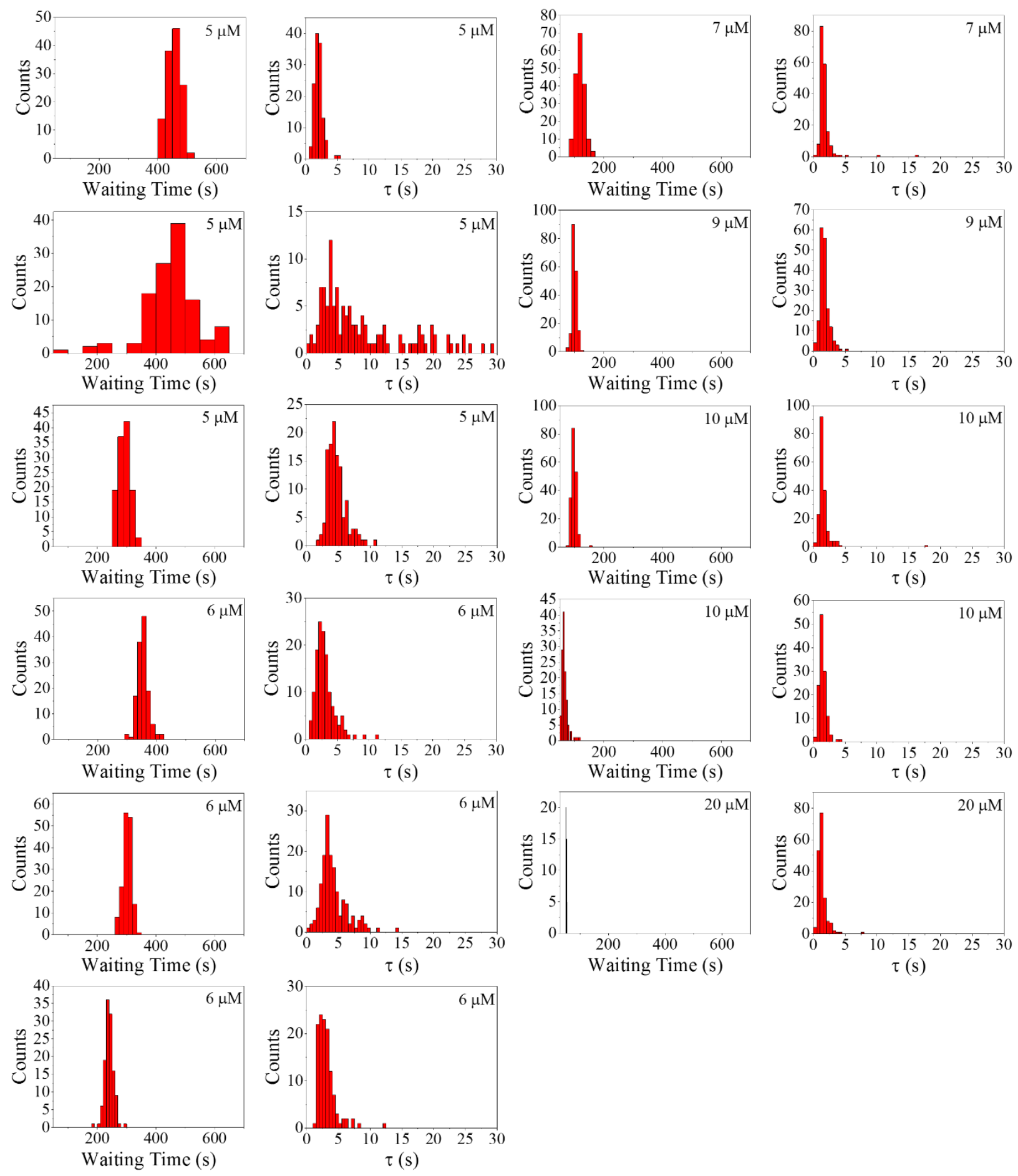

Figure S9. Histograms of waiting times and $\tau$ values for the entire set of $\mathrm{Au}(\mathrm{III}) \mathrm{Cl}_{3}$ concentration dependence experiments performed on $34 \mathrm{~nm}$ Ag nanospheres coated with 6MHA. Fits to these histograms were used to generate the data in Figure 3. 

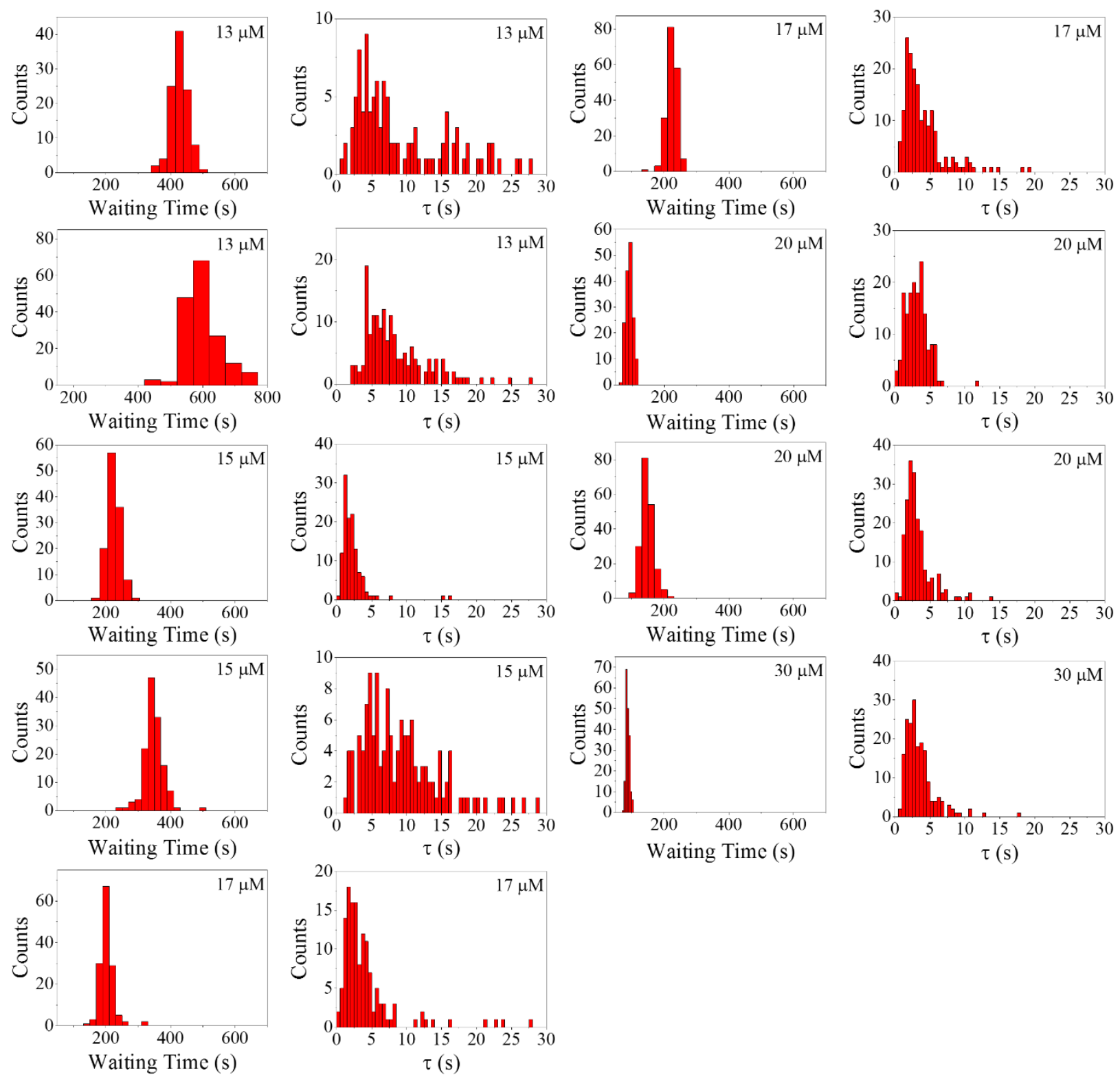

Figure S10. Histograms of waiting times and $\tau$ values for the entire set of $\mathrm{Au}(\mathrm{III}) \mathrm{Cl}_{3}$ concentration dependence experiments performed on $34 \mathrm{~nm} \mathrm{Ag}$ nanospheres coated with 11MUA. Fits to these histograms were used to generate the data in Figure 3. 

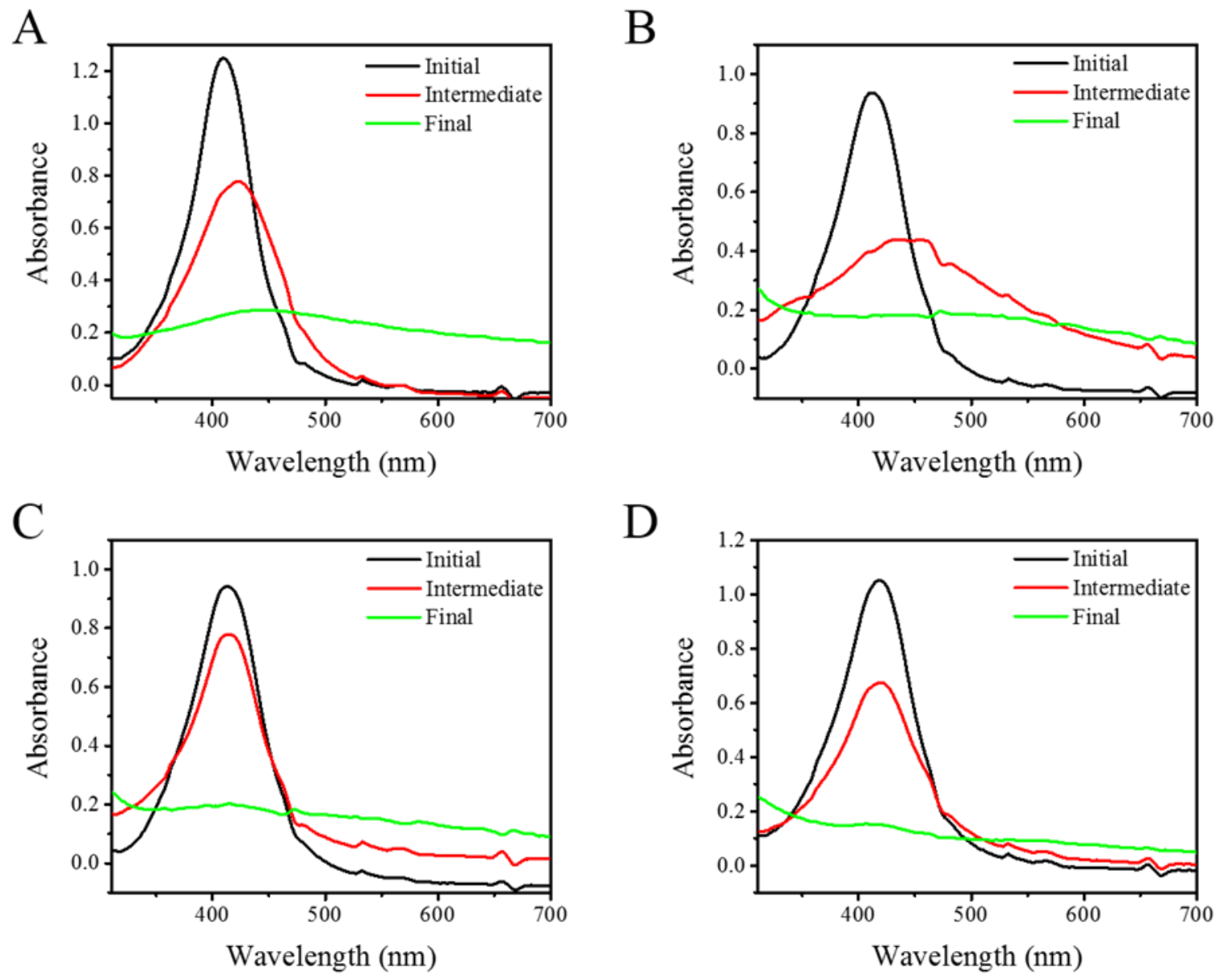

Figure S11. Ensemble, solution-phase galvanic exchange experiments for structural characterization. UV-Vis spectra of $34 \mathrm{~nm} \mathrm{Ag} \mathrm{nanospheres} \mathrm{coated} \mathrm{with} \mathrm{Na}_{3}$ Citrate (A), 3-MPA (B), 6-MHA (C) and 11-MUA (D) in their initial state (black curve), at an intermediate state of galvanic exchange with $\mathrm{Au}(\mathrm{III}) \mathrm{Cl}_{3}$ (red curve), and at the end of the titration with $\mathrm{Au}(\mathrm{III}) \mathrm{Cl}_{3}$ (green curve). A) $\mathrm{Na}_{3}$ Citrate coated nanoparticles exposed to 0,3 , and $25 \mathrm{nmol}$ of $\mathrm{Au}(\mathrm{III}) \mathrm{Cl}_{3}$ (black, red, and green respectively). B) 3-MPA coated particles exposed to 0, 20, and $45 \mathrm{nmol}$ of $\mathrm{Au}(\mathrm{III}) \mathrm{Cl}_{3}$ (black, red, and green respectively). C) 6-MHA coated nanoparticles exposed to 0, 30, and $55 \mathrm{nmol}$ of $\mathrm{Au}(\mathrm{III}) \mathrm{Cl}_{3}$ (black, red, and green respectively). D) 11-MUA coated nanoparticles exposed to 0,30 , and $100 \mathrm{nmol} \mathrm{Au}(\mathrm{III}) \mathrm{Cl}_{3}$ (black, red, and green respectively). The nanostructures in their final state were used to prepare samples for HAADF-STEM imaging and EDS analysis (Figures 4 and S12). Characterization of those in the intermediate state is shown in Figure 5. 


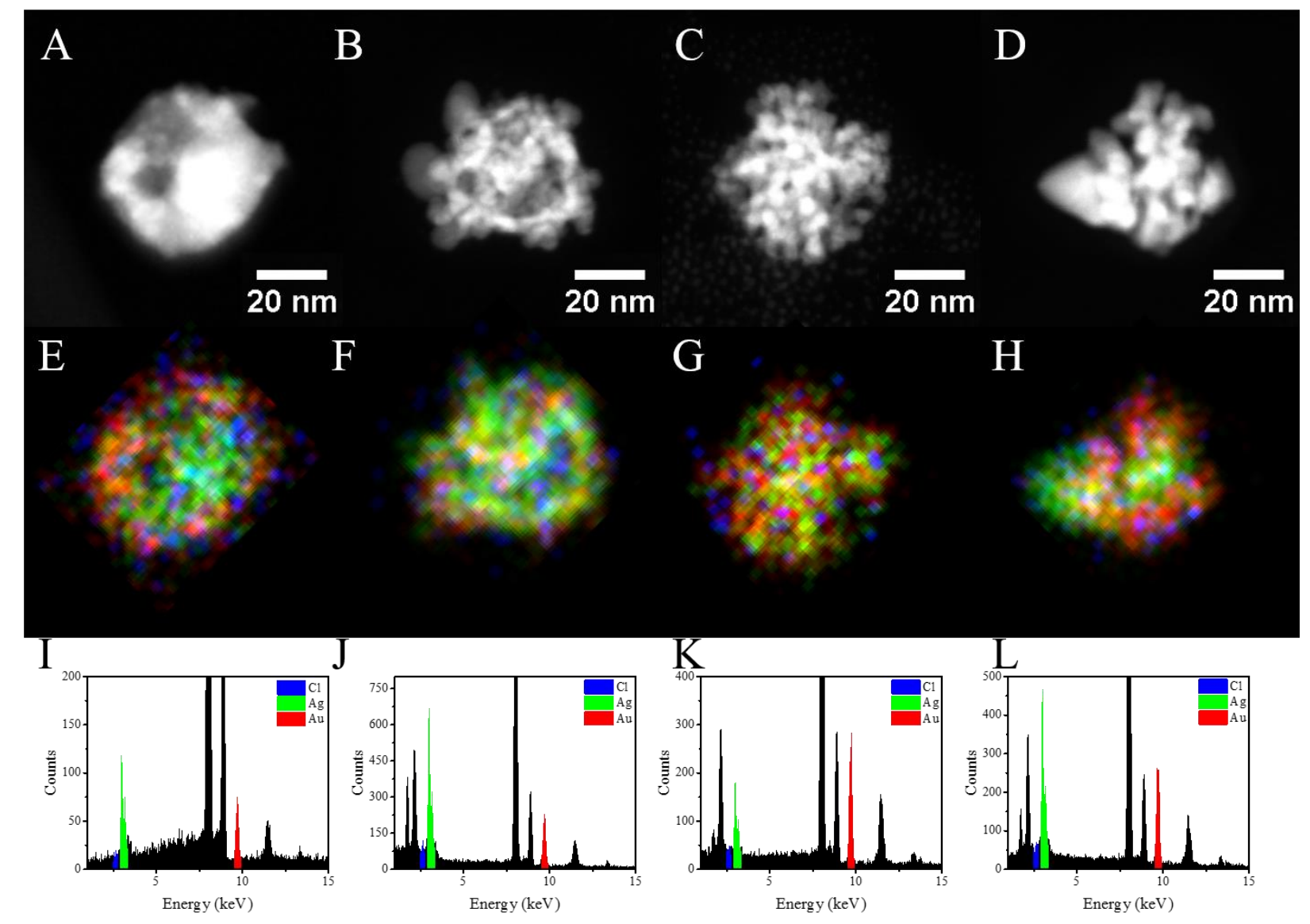

Figure S12. (Top) HAADF-STEM images, (middle) elemental maps and (bottom) EDS spectra of $\mathrm{Na}_{3}$ Citrate (A,E,I), 3-MPA (B,F,J), 6-MHA (C,G,K) and 11-MUA (D,H,L) coated nanoparticles following full exchange. $\mathrm{Au}$ is indicated by red, $\mathrm{Ag}$ in green, and $\mathrm{Cl}$ in blue. EDS spectra are averaged over the entire nanostructure. Weight ratios $(\% \mathrm{w} / \mathrm{w})$ for $\mathrm{Cl}: \mathrm{Ag}: \mathrm{Au}$ respectively are 2.4: 80.7: $16.8(\mathrm{~A}), 4.5: 85.2: 10.3(\mathrm{~B}), 5.3: 70.3: 24.4(\mathrm{C})$, and 3.7: 80.6: 15.7 (D), however, the average weight ratios of the wide-field of the entire sample (Figure 4), seen to be 3.3: 75.6: 21.0 (A), 2.1: 65.0: 32.9 (B), 3.2: 42.7: 54.1 (C), 2.8: 46.1: 51.0 (D) are likely to be more representative. On average, the nanostructures are found to contain quite substantial amounts of residual $\mathrm{Ag}$, however the relative amount of $\mathrm{Ag}$ is lower for longer chain ligands. This trend could potentially result from the larger amounts of $\mathrm{Au}(\mathrm{III}) \mathrm{Cl}_{3}$ added to achieve full exchange in longer ligand samples $(25,45,55$, and $100 \mathrm{nmol}$ respectively for A, B, C, and D), but we ensured that the latter is not the case. Although the nanoparticle solution from the titration, containing added $\mathrm{Au}(\mathrm{III}) \mathrm{Cl}_{3}$, was cast directly onto the TEM grid, the grid was washed with copious amounts of DI water before TEM imaging and EDS mapping to remove excess $\mathrm{Au}(\mathrm{III}) \mathrm{Cl}_{3}$ and other salts. 


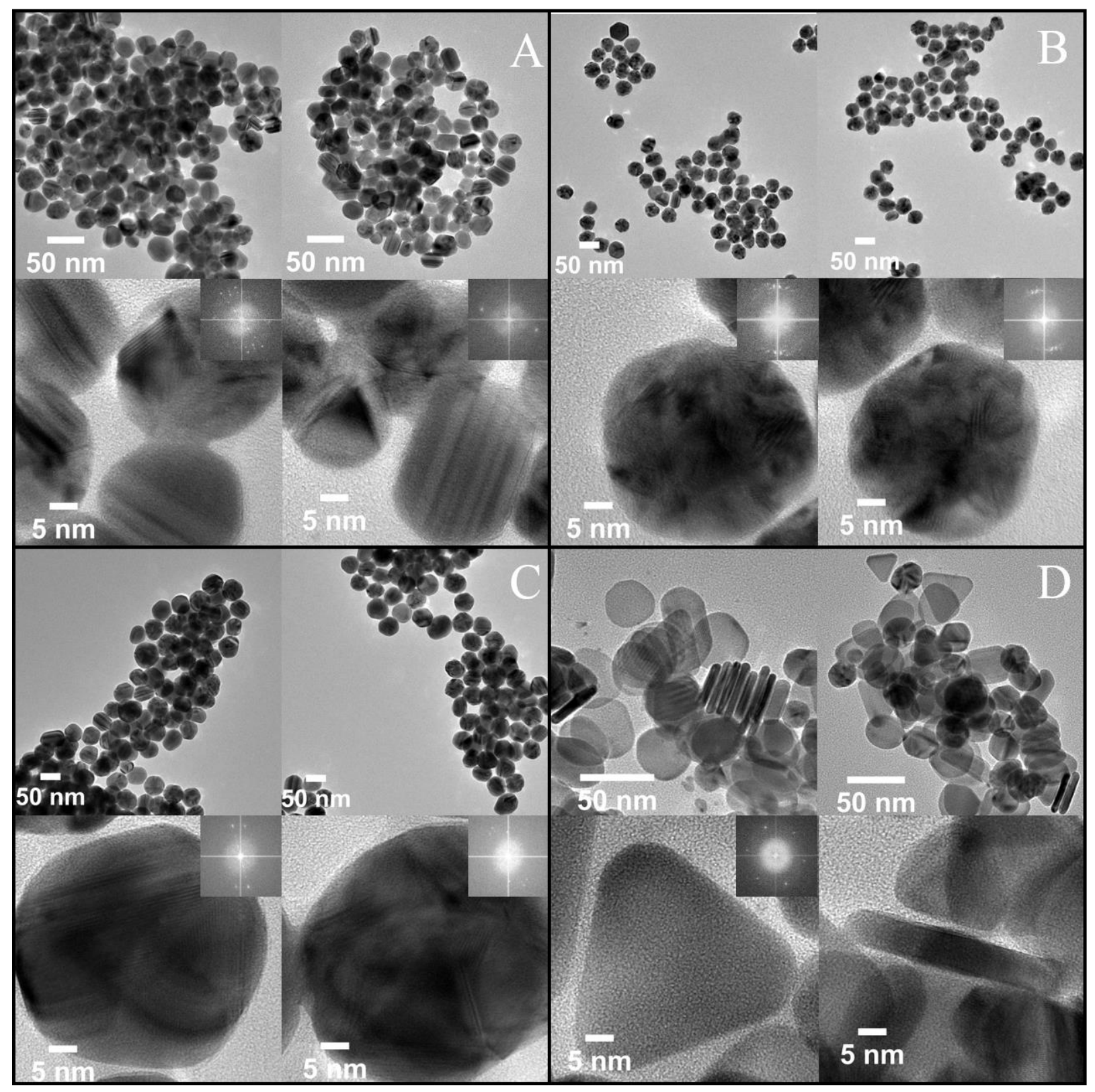

Figure S13. High-resolution transmission electron microscopy (HRTEM) images of $26 \mathrm{~nm}$ nanospheres (A), $34 \mathrm{~nm}$ nanospheres (B), $47 \mathrm{~nm}$ nanospheres (C) and nanoplates (D). Insets of the higher-magnification images show fast-Fourier transforms (FFTs) of the images. Reciprocal lattice spots seen in the FFTs indicate that the nanopsheres are predominantly polycrystalline, except for some of the $26 \mathrm{~nm}$ nanospheres, which are single-crystalline. On the other hand, the nanoplates are single crystalline, $5 \mathrm{~nm}$ thick on average, with their top flat surfaces comprised of the (111) facet of fcc Ag, and containing stacking faults perpendicular to their flat surface. 
A
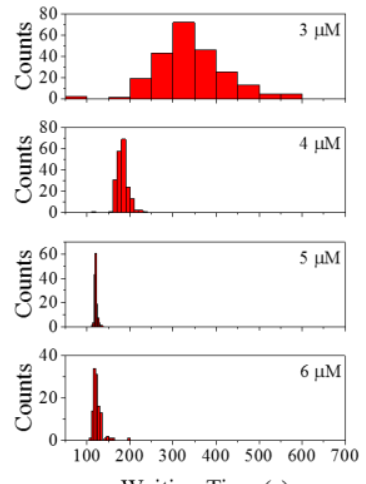

Waiting Time (s)

$\mathrm{C}$
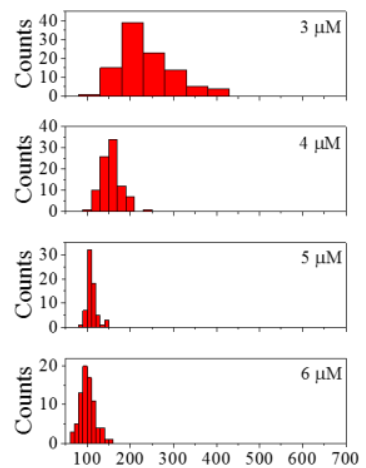

Waiting Time (s)
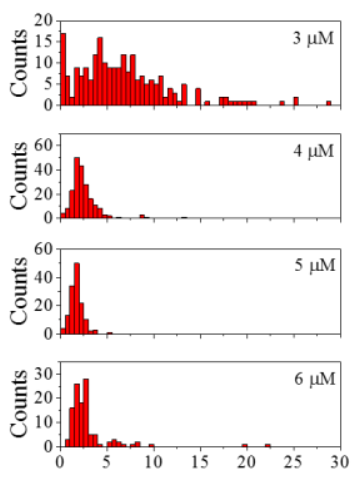

$\tau(\mathrm{s})$
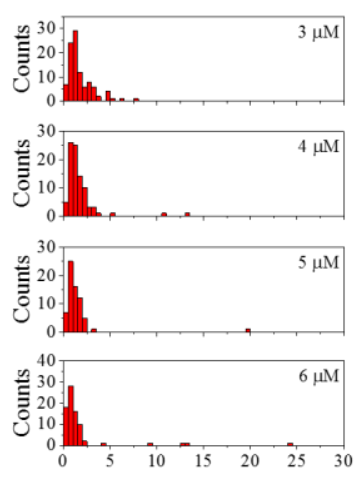

$\tau(\mathrm{s})$

B
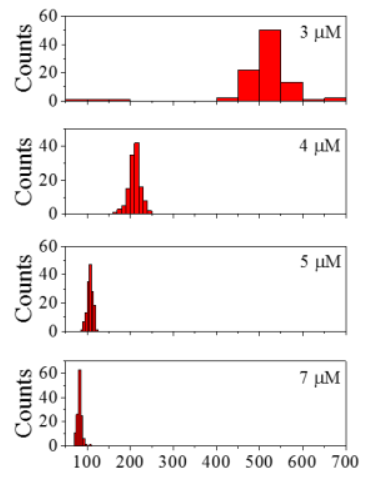

Waiting Time (s)

$\mathrm{D}$
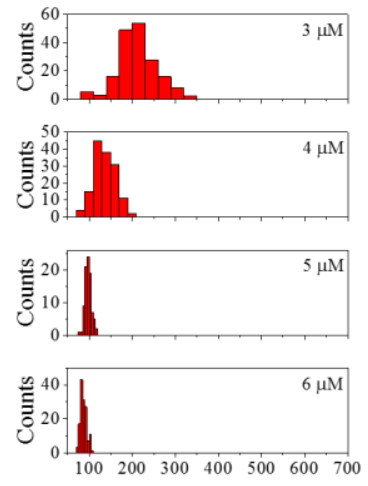

Waiting Time (s)
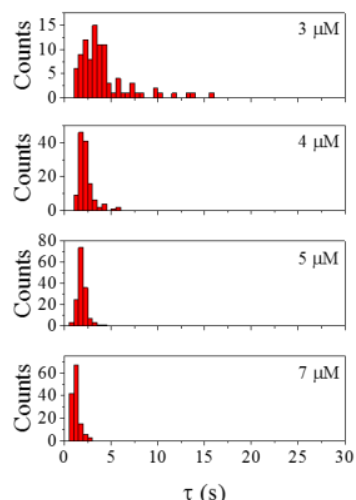

$\tau(\mathrm{s})$
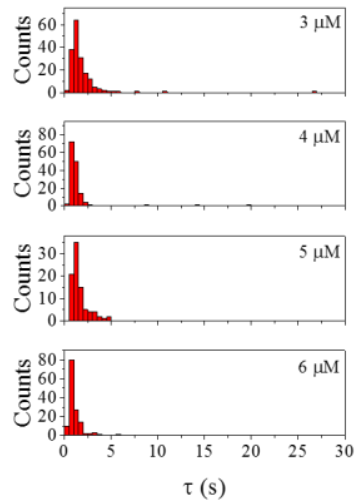

Figure S14. Histograms of waiting times and $\tau$ values for the entire set of $\mathrm{Au}(\mathrm{III}) \mathrm{Cl}_{3}$ concentration dependence experiments performed on $\mathrm{Na}_{3}$ citrate-coated $26 \mathrm{~nm}$ nanospheres (A), $34 \mathrm{~nm}$ nanospheres (B), $47 \mathrm{~nm}$ nanospheres (C) and nanoplates (D). Fits to these histograms were used to generate the data shown in Figure 6. 

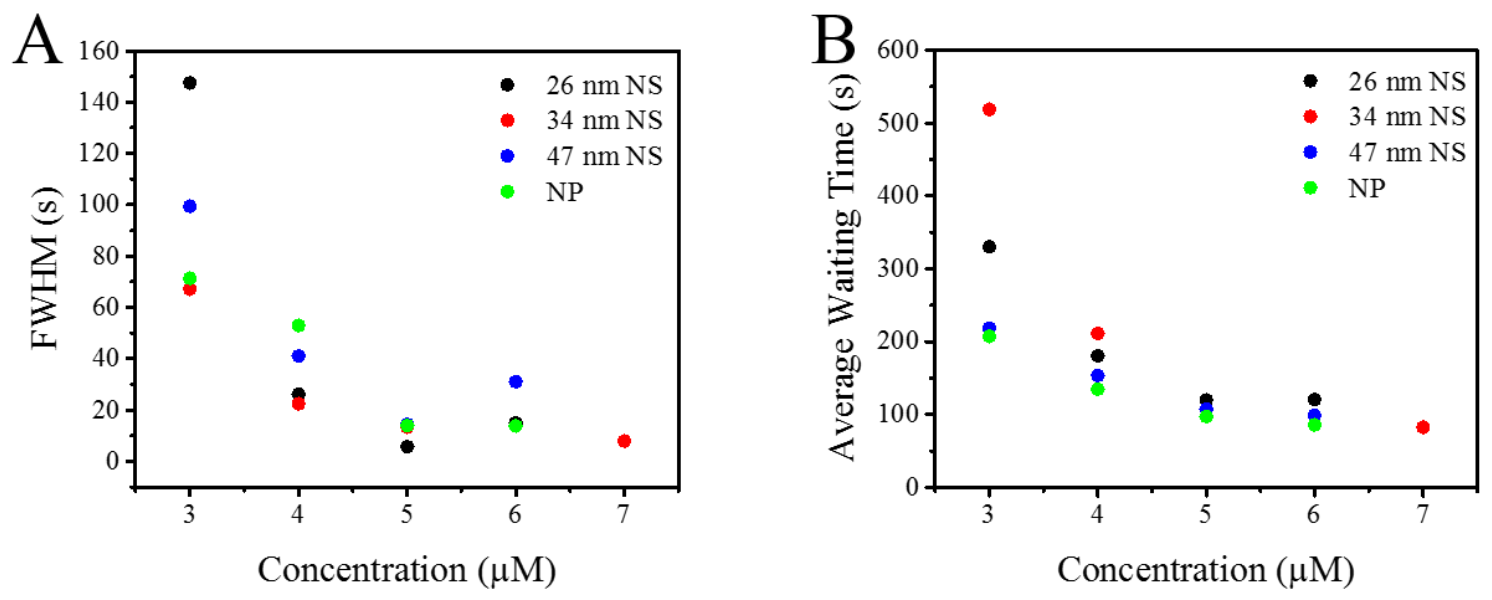

Figure S15. (A) FWHM and (B) average waiting time increase with decreasing concentration of $\mathrm{Au}(\mathrm{III}) \mathrm{Cl}_{3}$, following a similar trend irrespective of nanoparticle size or shape. Average waiting times are shown as a function of $\mathrm{Au}(\mathrm{III}) \mathrm{Cl}_{3}$ concentration for $\mathrm{Na}_{3}$ citrate-coated nanospheres of three different sizes and also $\mathrm{Na}_{3}$ citrate-coated nanoplates. All experiments were performed in DI water at a flow rate of $1.5 \mathrm{~mL} / \mathrm{hr}$. The acquisition time was set to $0.5 \mathrm{~s}$, except for the $26 \mathrm{~nm}$ nanosphere case, for which the acquisition time was set to $1.5 \mathrm{~s}$. The lamp power was set to a maximum, except for the $47 \mathrm{~nm}$ nanosphere, for which it was set to $33.41 \%$ of maximum fluency. The average waiting time was derived from the peak of the Gaussian fit of the waiting time distribution, as exemplified in Figure S2. Thus, we demonstrate that the average waiting time follows the same trend as a function of $\mathrm{Au}(\mathrm{III}) \mathrm{Cl}_{3}$ concentration as the trend of the FWHM. 


\section{Supporting Information Movie Captions}

SI Movie 1. Video microscopy of a wide-field of several $\mathrm{Na}_{3}$ citrate-coated single $\mathrm{Ag}$ nanoparticles undergoing a galvanic replacement reaction in response to $\mathrm{Au}\left(\mathrm{III} \mathrm{Cl}_{3}\right.$. Data from this video was used to construct Figure $1(\mathrm{G}, \mathrm{H}, \mathrm{I})$ in the main text. The bright scatterers, which represent individual Ag nanoparticles, undergo an abrupt conversion to dimmer scatterers, which representing $\mathrm{Au} / \mathrm{Ag}$ nanocages. This experiment was performed with a $\mathrm{Au}(\mathrm{III}) \mathrm{Cl}_{3}$ concentration of $6 \mu \mathrm{M}$, a lamp power of $12.11 \%$, and a flow rate of $1.5 \mathrm{~mL} / \mathrm{hr}$. The $\mathrm{Au}^{3+}$ solution entered the cell at $\mathrm{t}=20 \mathrm{~s}$. Movies are shown sped up at $50 \mathrm{fps}$. The time is indicated in seconds at the bottom, right corner. This video was collected on an Olympus IX 51 microscope using a PyLoN CCD camera. The pulsations seen in the video are caused by the action of the syringe pump used to flow the solution.

SI Movie 2. Video microscopy of a wide-field of several 3-MPA-coated single Ag nanoparticles undergoing a galvanic replacement reaction in response to $\mathrm{Au}(\mathrm{III}) \mathrm{Cl}_{3}$. Data from this video was used to construct Figure 1 (D, E, F) in the main text. The bright scatterers, which represent individual Ag nanoparticles, undergo an abrupt conversion to dimmer scatterers, which representing $\mathrm{Au} / \mathrm{Ag}$ nanocages. This experiment was performed with a $\mathrm{Au}(\mathrm{III}) \mathrm{Cl}_{3}$ concentration of $6 \mu \mathrm{M}$, a lamp power of $12.11 \%$, and a flow rate of $1.5 \mathrm{~mL} / \mathrm{hr}$. The $\mathrm{Au}^{3+}$ solution entered the cell at $\mathrm{t}=20 \mathrm{~s}$. Movies are shown sped up at $50 \mathrm{fps}$. The time is indicated in seconds at the bottom, right corner. This video was collected on an Olympus IX 51 microscope using a PyLoN CCD camera. The pulsations seen in the video are caused by the action of the syringe pump used to flow the solution.

SI Movie 3. Video microscopy of a wide-field of several 6-MHA-coated single Ag nanoparticles undergoing a galvanic replacement reaction in response to $\mathrm{Au}(\mathrm{III}) \mathrm{Cl}_{3}$. Data from this video was used to construct Figure $1(\mathrm{~A}, \mathrm{~B}, \mathrm{C})$ in the main text. The bright scatterers, which represent individual Ag nanoparticles, undergo an abrupt conversion to dimmer scatterers, which representing $\mathrm{Au} / \mathrm{Ag}$ nanocages. This experiment was performed with a $\mathrm{Au}(\mathrm{III}) \mathrm{Cl}_{3}$ concentration of $6 \mu \mathrm{M}$, a lamp power of $12.11 \%$, and a flow rate of $1.5 \mathrm{~mL} / \mathrm{hr}$. The $\mathrm{Au}^{3+}$ solution entered the cell at $\mathrm{t}=20 \mathrm{~s}$. Movies are shown sped up at $50 \mathrm{fps}$. The time is indicated in seconds at the bottom, right corner. This video was collected on an Olympus IX 51 microscope using a PyLoN CCD camera. The pulsations seen in the video are caused by the action of the syringe pump used to flow the solution.

SI Movie 4. Video microscopy of a wide-field of several 11-MUA-coated single Ag nanoparticles undergoing a galvanic replacement reaction in response to $\mathrm{Au}(\mathrm{III}) \mathrm{Cl}_{3}$. Data from this video is referenced in the main text, in the caption for Figure 1. The bright scatterers, which represent individual Ag nanoparticles, undergo an abrupt conversion to dimmer scatterers, which representing $\mathrm{Au} / \mathrm{Ag}$ nanocages. This experiment was performed with a $\mathrm{Au}(\mathrm{III}) \mathrm{Cl}_{3}$ concentration of $6 \mu \mathrm{M}$, a lamp power of $12.11 \%$, and a flow rate of $1.5 \mathrm{~mL} / \mathrm{hr}$. The $\mathrm{Au}^{3+}$ solution entered the cell at $\mathrm{t}=27 \mathrm{~s}$. Movies are shown sped up at $50 \mathrm{fps}$. The time is indicated in seconds at the bottom, right corner. This video was collected on an Olympus IX 51 microscope using a PyLoN CCD camera. The pulsations seen in the video are caused by the action of the syringe pump used to flow the solution. 


\section{Supporting Information References}

(1) Smith, J. G.; Jain, P. K. submitted 2016. 